\title{
Panoramic Radiographic Analysis of Signs of Proximity to the Third Molar Roots of the Mandibular Canal in Yemen
}

\author{
Journal of Medical Clinical Case Reports
}

Research Article

Nashwan Yahya A Al-Shamahi' ${ }^{1}$ MD, PhD, Al-Kasem Mohammed Abbas ${ }^{2}$ BDS, MSc. PhD, Hassan Abdulwahab Al-Shamahy ${ }^{3 *}$ MD, PhD, Ameen Abdullah Yahya Al-Akwa ${ }^{4}$ BDS, MSc. PhD, Khaled A AL-Haddad ${ }^{4}$ BDS, MSc. PhD, Khaled M Al-Ghaffari ${ }^{2}$ BDS, MSc., Ammar M Al-Mortada ${ }^{2}$ BDS, MSc. and Mohammed A Al-labani ${ }^{4}$ BDS, MSc. PhD

\author{
${ }^{1}$ Medicine Department, Faculty of Medicine and Health \\ Sciences, Sana'a University, Republic of Yemen.
}

${ }^{2}$ Department of Maxillo-Facial, Faculty of Dentistry, Sana'a University, Republic of Yemen.

${ }^{3}$ Departement of Basic Sciences, Faculty of Dentistry, Sana'a University, Republic of Yemen

${ }^{4}$ Orthodontics, Pedodontics and Prevention Department Faculty of Dentistry, Sana'a University, Yemen.

\author{
*Correspondence author \\ Prof. Hassan A. Al-Shamahy \\ Faculty of Dentistry \\ Sana'a University \\ Yemen
}

Submitted : 11 Nov 2020 ; Published : 27 Nov 2020

\begin{abstract}
Objectives: The objectives of this study were to assess the relationship between the third molar and the mandibular canal, to identify the radiographic markers most prevalent in predicting connectivity between these structures, and to associate these signs and proximity with the position of the third molar according to Bell, Gregory's and Winter's classifications.

Subjects and methods: A retrospective cross-sectional study was conducted in the city of Sana'a on a sample of Yemeni patients in Ammar Dental Clinics who underwent panoramic radiography in the year 2019 until September 2020. The study consisted of panoramic radiographs of 597 patients with third molars with a total of 1017 third molars; the number of males was 216 (36.2\%) and 381 females (63.8\%). The panoramic radiographs were evaluated for proximity signs. Inclusion criteria were patients of both sexes who had at least a fully formed third molar of the mandible that was adjacent to the second molar. The radiographs were excluded if evidence of cystic, neoplastic, or extensive caries processes was detected. Seven radiological signs were used to determine if there was contact between the third molar and the mandibular canal. Results: A proximity to the mandibular canal was verified for 620 (61\%) of 1017 third molars. The most frequent radiographic signs of proximity were darkened roots (315 teeth [31\%]) and discontinuity of the mandibular canal (267 teeth [26.3\%]). Third molar/mandibular canal proximity was found significantly more frequently in female patients and in patients aged 24 years and younger $(P<0.001)$. The tooth positions most frequently associated with proximity between the third molar and the mandibular canal were position C (highest point of the third molar located at or below the cervical margin of the second molar) and the mesioangular position (long axis of the third molar angled mesially toward the second molar). Conclusions: The frequency of third molar/mandibular canal proximity was greater in female patients and patients aged 24 years or younger. The most frequently observed signs of proximity were darkening of the roots and discontinuity of the mandibular canal. The tooth positions most frequently associated with proximity of the third molar to the mandibular canal were position $C$ and the mesioangular position.
\end{abstract}

Keywords: mandibular nerves, panoramic radiographs, third molar, signs of proximity

\section{Introduction}

It is known that the third molars are the last teeth to erupt and their root formation usually begins around the age of 15 years and erupts in about 20 years [1-5]. One of the common practices in dental clinics is the surgical removal of the third molar of the lower jaw [6,7]. According to published researches, the third molars are the teeth with the highest frequency of impaction, followed by the canines [8-10]. Several factors contribute to the impact of the third molar, including the length of the arch, the teeth size and position of the adjacent second molar, bone density, eruption path, and the developmental limit of the mandible [11,12]. The modern diet was also found to enhance the effect by decreasing the effort required to chew, provided that little motivation for the growth of the upper jaw; as a result, modern humans have a higher rate of dental impactions than humans in the recent past [12]. Third molar impaction may lead to pericarditis, necrotic lesions, abscesses, dental tumors, gum disease, and external root resorption of adjacent teeth $[5,9,12-15]$.

When previous unfavorable conditions arise, the third molar 
excision is recommended surgically. Preventive surgery may also be indicated, but the surgeon must balance the surgical risks associated with removal, such as injury to the inferior alveolar nerve (IAN), against the potential consequences of not removing the tooth, the most important of which is the risk of fractures of the mandible [16,17]. Careful surgical planning and a deep knowledge of anatomy are crucial for the removal of the third molar due to its close association with vital structures such as the IAN, the lingual nerve and the adjacent second molar [16]. It has been reported from Sarikov and Juodzbalys [16] that the frequency of IAN injury during the removal of the third molar varies from $0.35 \%$ to $8.4 \%$ [13]. The damage resulted from direct pressure on the nerve or indirect forces transmitted by the root during the elevator extraction $[16,18]$.

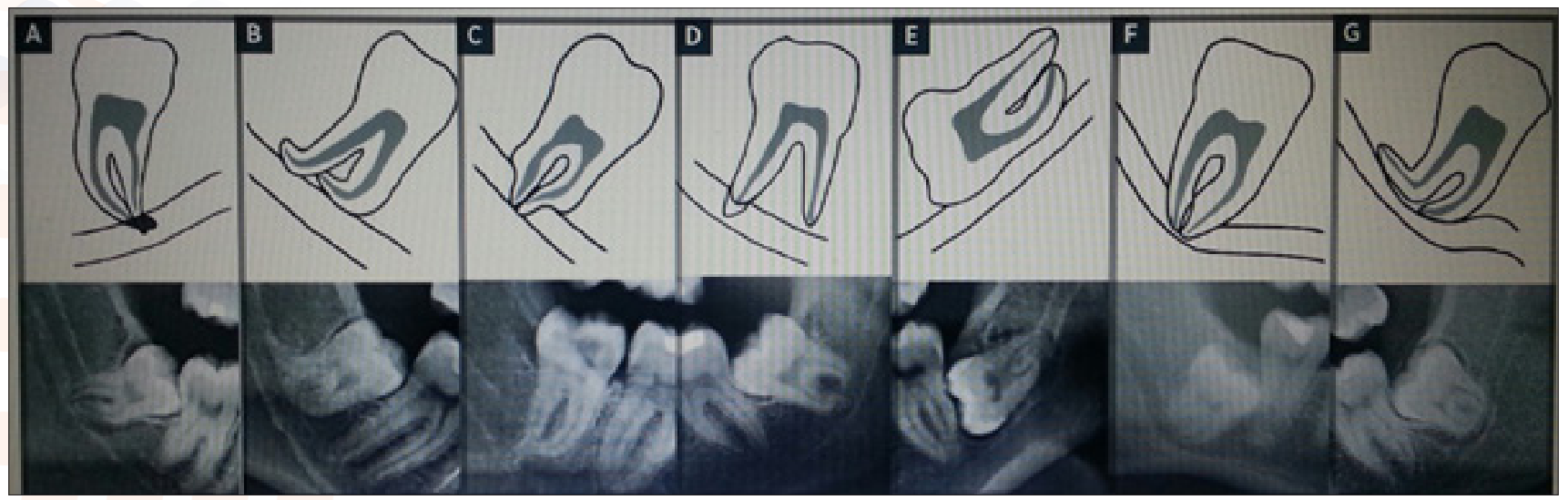

Figure 1:Radiographic signs of proximity relating the third molar and the mandibular canal. A. Darkening of the roots. B. Deviation of the roots. C- Narrowing of the roots. D. Dark and cleft crest. E- Interruption of the maxillary canal. And the. Deviation of the mandibular canal. G - Narrowing of the lower canal (from Kim et al.[25].)

Rood and Shehab identified some radiographic markers associated with the connection between the third molars and the mandibular canal [19]. These signs are the deflection of the roots, the darkening of the roots, narrowing of the roots, a dark and bifid apex, interruption of the mandibular canal, deflection of the mandibular canal, and narrowing of the mandibular canal (Fig. 1) [19]. The evaluation of these markers is very important for better surgical planning, thus helping the surgeon avoid IAN injuries. Panoramic radiography is an effective preoperative evaluation of the lower third molars, and these images are widely used to assess the spread of the impaction and the proximity of the third molar to the maxillary canal. [20,21]. Also, its main advantage over other radiological examinations is that it reduces the time of exposure to radiation $[21,22]$.

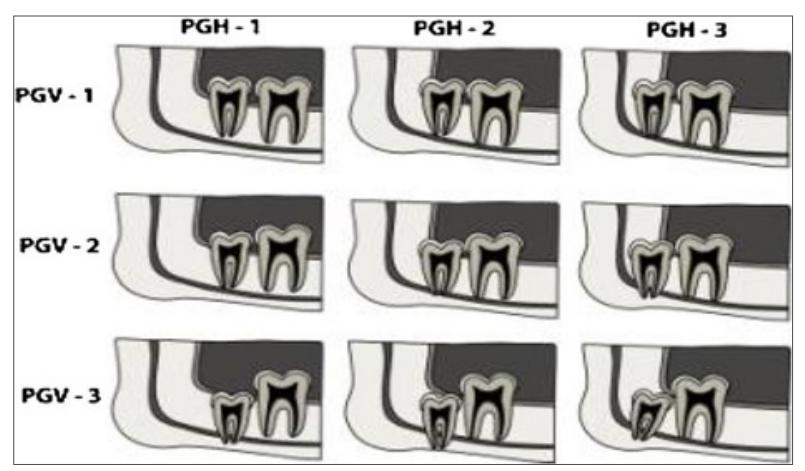

Figure 2: Pell and Gregory classification of the lower third molars. Horizontal classification PGH-1: normal apical region, PGH-2: moderate apical region, PGH-3: small apical region. Vertical classification: $A=P G V-1$ : the occlusal level of the third molar at the same level as the occlusal level of the second molar, B = PGV-2: the occlusal level of the third molar lies between the occlusal plane and the cervical edge of the second molar, $\mathrm{C}=\mathrm{PGV}-3$ : the occlusal level of the third molar lies below the cervical margin of the second molar. Regarding the occlusal level, the affected third molar is in position A when its highest point is in the plane with or above occlusal level, position $\mathrm{B}$ when its highest point is between the occlusal plane and the cervical edge of the second molar, and position $\mathrm{C}$ when it is the highest point at Or below the cervical margin of the second molar [19,23].

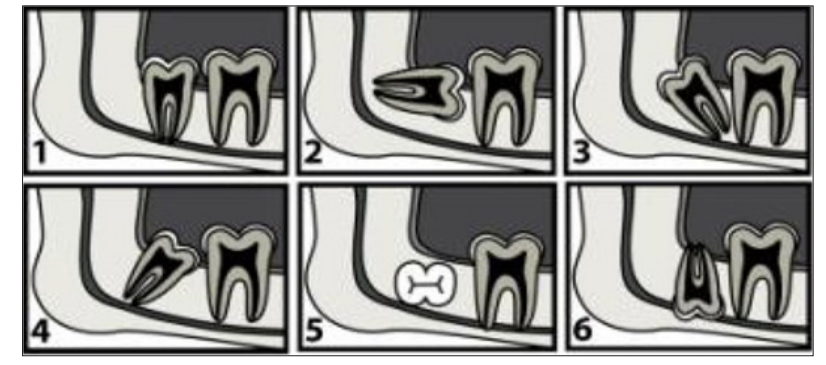

Figure 3: Winter's classification: Third molars are classified according to their inclination to the long axis of the second molar. (1) vertical angulation, (2) horizontal angulation, (3) distoangular angulation, (4) mesioangular angulation, (5) transversal angulation, (6) inverse angulation. The classification proposed by Winter's evaluates the long axis of the embedded third molar in relation to the long axis of the second molar [24] According to this classification, the third molar may be found in the following positions: vertical, when the long axis of the third molar is parallel to the second molar; mesioangular, when the long axis of the third molar is angled mesially toward the second molar; distoangular, when the long axis of the third molar is angled distally away from the second molar; horizontal, when the long axis of the third molar is perpendicular to the long axis of the second molar; linguoversion, when the long axis of the third molar is angled 
lingually in relation to the second molar; buccoversion, when the long axis of the third molar is angled buccally in relation to the second molar; and inversion, when the crown of the third molar is oriented in the direction of the mandibular border and the root is oriented to the occlusal plane.[24]

The present study aimed to assess the association between the third molar and the mandibular canal, to identify the most common radiographic markers in predicting the contact between the two structures, and to correlate these signs and proximity with the position of the third molar according to Pell and Gregory's (Fig. 2) and Winter's (Fig 3) classifications $[19,23,24]$.

\section{Subjects and Methods}

A retrospective cross-sectional study was carried out in the city of Sana'a on a sample of Yemeni patients in Ammar Dental Clinics who underwent panoramic radiography in the year 2019 until September 2020. The study consisted of panoramic radiographs of 597 patients with third molars with a total of 1017 third molars; the number of males was 216 (36.2\%) and 381 females $(63.8 \%)$. The panoramic radiographs were evaluated for proximity signs. Inclusion criteria were patients of both sexes who had at least a fully formed third molar of the mandible that was adjacent to the second molar. The radiographs were excluded if evidence of cystic, neoplastic, or extensive caries processes was detected. Seven radiological signs were used to determine if there was contact between the third molar and the mandibular canal. All panoramic radiographs were made with a Proline XC. By the first investigator these images were carefully examined.

Lighting conditions with free use of tools in photo software, such as brightness and contrast. The seven radiotracers described by Rood and Shehab were used to analyze the contact of the third molar with the lower canal (Fig. 1) [19, 25]:

1. Darkening of the roots. When the roots and the lower canal are in contact, a loss of density is observed due to a decrease in the amount of tooth structure or the cortical lining of the mandibular canal.

2. The deflection of the roots. There is a sudden change in the root pathway when it reaches the mandibular canal.

3. Narrowing of the roots. The canal of the mandible and the roots of the third molar are overlapping or in contact, indicating that the narrowed region is surrounded by the canal.

4. Dark and bifid apex. When the apex of the third molar crosses the mandibular canal, it results in a radiating appearance or cleft due to the shadow of the periodontal ligament.

5. Lower canal disconnection. Detachment is characterized by a disconnection of the roof or floor of the mandibular canal (shaded lines) at the location of the third molar

6. Deflection of the mandibular canal. A change in the direction of the lower canal is observed upon the passage of the roots of the third molar.

7. Mandibular canal stenosis. This is the narrowing of the duct diameter when the canal and the roots of the third molar are in contact or overlapping. The presence of proximity between the third molar and the mandibular canal was determined on a yes / no basis. Each third molar was assessed individually if there was more than 1 on the radiograph. The examiners scored a "yes" decision regarding proximity if any of the seven radiographic signs were present.

The evaluators used the Pell and Gregory's classifications (Fig. 2 ) and Winter's (Fig. 3) to identify and record the position of the third molars $[23,24]$. The radiographs were evaluated by the examiner who is the first author. Pell and Gregory classified (Fig.2) [23] mandibular third molars based on the relationships of the impacted tooth to the anterior border of the mandibular ramus (class) and the occlusal plane (position). In relation to the mandibular ramus, the third molar is class I when the space between the anterior border of the ramus and the distal surface of the second molar is greater than the mesiodistal parameter of the impacted third molar crown; class II when this space is smaller than the mesiodistal diameter of the impacted crown; and class III when the ramus is adjacent to the second molar, meaning that the third molar is situated totally within the ramus. As for occlusal level, the affected third molar is in position A when its highest point is in the plane with or above occlusal level, and position $\mathrm{B}$ when its highest point is between the occlusal plane and the cervical edge of the second molar, and position $\mathrm{C}$ when it is the highest point at Or just below the cervical margin of the second molar. The classification proposed by Winter (Fig.3) [24] evaluates the long axis of the embedded third molar in relation to the long axis of the second molar. According to this classification, the third molar may be found in the following positions: vertical, when the long axis of the third molar is parallel to the second molar; mesioangular, when the long axis of the third molar is angled mesially toward the second molar; distoangular, when the long axis of the third molar is angled distally away from the second molar; horizontal, when the long axis of the third molar is perpendicular to the long axis of the second molar; linguoversion, when the long axis of the third molar is angled lingually in relation to the second molar; buccoversion, when the long axis of the third molar is angled buccally in relation to the second molar; and inversion, when the crown of the third molar is oriented in the direction of the mandibular border and the root is oriented to the occlusal plane.

\section{Ethical Approval}

The Medical Ethics and Research Committee at the Faculty of Medicine and Health Sciences at Sana'a University gave us ethical approval. The patient identification and $\mathrm{x}$-ray were kept confidential. 


\begin{tabular}{|l|l|l|}
\hline Radiographic sign & Number & Percentage \\
\hline A-Darkening of the roots & 315 & 31 \\
\hline B-Deflection of the roots & 11 & 1.08 \\
\hline C-Narrowing of the roots & 3 & 0.29 \\
\hline D-Dark and bifid apex & 6 & 0.59 \\
\hline E-Discontinuity of the mandibular canal & 267 & 26.3 \\
\hline F-Deflection of the mandibular canal & 6 & 0.59 \\
\hline G-Narrowing of the mandibular canal & 12 & 1.18 \\
\hline Total & $620 / 1017$ & 61 \\
\hline
\end{tabular}

Table 1: Frequency of radiographic signs of third molar/mandibular canal proximity ( $\mathrm{N}=1017$ teeth, Fig.1).

\begin{tabular}{|l|l|l|l|l|l|}
\hline \multirow{2}{*}{ Group } & \multicolumn{2}{|l|}{ Positive TM/MP } & \multirow{2}{*}{ CI 95\% } & OR & P* \\
\cline { 2 - 5 } & No & $\%$ & & \\
\hline Sex & 140 & 64.8 & $0.47-0.97$ & 0.68 & $0.036^{*}$ \\
\hline Male $\mathrm{n}=216$ & 278 & 73 & $1.1-2.1$ & 1.5 & $0.036^{*}$ \\
\hline Female $\mathrm{n}=381$ & 261 & 81 & $2.2-4.6$ & 3.2 & $<0.0001^{*}$ \\
\hline Age group & 140 & 65 & $0.4-1.0$ & 0.69 & $0.044^{*}$ \\
\hline 15-24 years $\mathrm{n}=322$ & 17 & 28.3 & $0.07-0.24$ & 0.13 & $<0.0001^{*}$ \\
\hline $25-34$ years $\mathrm{n}=215$ & 418 & 70 & & & \\
\hline$\geq 35$ years $\mathrm{n}=60$ & & & & & \\
\hline Total $\mathrm{n}=597$ &
\end{tabular}

Table 2: The rate of third molar/mandibular canal proximity (TM/MP) in association with patient sex and age $(\mathrm{N}=597$ patients). *Uncorrected chi-square test

\begin{tabular}{|l|l|l|l|l|l|l|l|l|l|l|l|}
\hline Proximity & IA & IIA & IIIA & IB & IIB & IIIB & IC & IIC & IIIC & TOTAL & $p^{\text {a }}$ \\
\hline Yes & 352 & 153 & 2 & 59 & 35 & 1 & 7 & 10 & 1 & 620 & $<0.001^{*}$ \\
& $(59.7 \%)$ & $(88.4 \%)$ & $(75 \%)$ & $(90.7 \%)$ & $(20.9 \%)$ & $(100 \%)$ & $(87.5 \%)$ & $(100 \%)$ & $(100 \%)$ & $(61 \%)$ & \\
\hline \multirow{2}{*}{ No } & 237 & 20 & 1 & 6 & 132 & 0 & 1 & 0 & 0 & 397 \\
& $(40.2 \%)$ & $(11.6 \%)$ & $(25 \%)$ & $(9.3 \%)$ & $(79.1 \%)$ & $(0.0 \%)$ & $(12.5 \%)$ & $(0.0 \%)$ & $(0.0 \%)$ & $(39 \%)$ & \\
\hline \multirow{2}{*}{ Total } & 589 & 173 & 3 & 65 & 167 & 1 & 8 & 10 & 1 & 1017 & \\
& $(57.9 \%)$ & $(17 \%)$ & $(0.29 \%)$ & $(6.4 \%)$ & $(16.4 \%)$ & $(0.09 \%)$ & $(0.78 \%)$ & $(0.98 \%)$ & $(0.09 \%)$ & $(100)$ & \\
\hline
\end{tabular}

$a=$ Pearson chi-square test.

Table 3: Frequency (\%) of Pell and Gregory's classification categories according to third molar/mandibular canal proximity (N $=1017$ teeth) (Fig 2).

\begin{tabular}{|l|l|l|l|l|l|l|l|l|}
\hline Proximity & Vertical & Mesioangular & Distoangular & Horizontal & Transalveolar & Inverse & TOTAL & $\mathrm{p}^{\mathrm{a}}$ \\
\hline Yes & 327 & 190 & 4 & 59 & 40 & 0 & 620 & $<0.001^{*}$ \\
& $(53.2 \%)$ & $(76 \%)$ & $(66.7 \%)$ & $(71.1 \%)$ & $(63.5 \%)$ & $(0.0 \%)$ & $(61 \%)$ & \\
\hline No & 288 & 60 & 2 & 21 & 23 & 0 & 397 & \\
& $(46.8 \%)$ & $(24 \%)$ & $(33.3 \%)$ & $(28.9 \%)$ & $(36.5 \%)$ & $(0.0 \%)$ & $(39 \%)$ & \\
\hline Total & 615 & 250 & 6 & 83 & 63 & 0 & 1017 & \\
& $(60.5 \%)$ & $(24.6 \%)$ & $(0.59 \%)$ & $(8.2 \%)$ & $(6.2 \%)$ & $(0.0 \%)$ & $(100)$ & \\
\hline
\end{tabular}

$a=$ Pearson chi-square test.

Table 4: Frequency (\%) of Winter's classification categories according to third molar/mandibular canal proximity $(\mathrm{N}=1017$ teeth) (Fig 3). 
Proximity to the mandibular canal was confirmed for 620 (61\%) of 1017 third molars. The most common radiographic signs of proximity were darkened roots (315 teeth [31\%]) and discontinuity of the mandibular canal (267 teeth [26.3\%], whereas deflection of the roots $(1.08 \%)$, narrowing of the roots $(0.29 \%)$, dark and bifid apex $(0.59 \%)$, deflection of the mandibular canal $(0.59 \%)$, and narrowing of the mandibular canal $(1.18 \%)$ were rare. When we consider the rate of molar / mandibular canal proximity (TM / MP) in conjunction with the sex and age of the patient, there was a significant association between TM / MP with female patients where the TM / MP rate was $73 \%$, with an associated odds ratio of 1.5 , CI95\%: $1.1--2.1(p=0.036)$ (Table 2). Also for age groups, there was a significant association between TM / MP with the younger age group (15-24 years) as TM / MP was $81 \%$, with an associated odds ratio of 3.2, 95\% CI: $2.2-4.6$ (p <0.0001). ) (Table 2). Table 3 shows the frequency (\%) of Bell \& Gregory's classification classes according to third molar / mandibular proximity (Fig.2). IA was predominant with 589 teeth $(57.9 \%)$ 352 (59.7\%) with TM / MP, followed by IIA with 173 teeth (17\%) 153 (88.4\%) with TM / MP, IIB with 167 teeth (16.4\%) $35(20.9 \%)$ had TM / MP and IB with 65 teeth (6.4\%) 59 (90.7\%) had TM / MP. However, other situations were less frequent and occurred less than $1 \%$ (Table 3). Table 4 shows the frequency (\%) of Winter's classification classes according to third molar / mandibular proximity (Fig.3). Vertical position was predominant with 615 teeth $(60.5 \%) 327(53.2 \%)$ of them with TM / MP, followed by mesioangular with 250 teeth (24.6\%) $190(76 \%)$ with TM / MP, horizontal with 83 teeth. $(8.2 \%) 59(71.1 \%)$ with TM / MP and trans-alveolar with 63 $(6.3 \%) 40(63.5 \%)$ suffering from TM / MP. However, other situations were less frequent and occurred less than $1 \%$ (Table 3).

\section{Discussion}

The impact rate of the mandibular third molars is high, and it requires careful radiological interpretation and a high level of preparation by the surgeon in order to carry out removal procedures without causing IAN injuries and the consequent paresthesia. [26]. In the current study; Proximity to the mandibular canal was confirmed for 620 (61\%) of 1017 third molars, and the most common radiographic signs of proximity were dark roots (315 teeth [31\%]) and discontinuity of the mandibular canal (267 teeth [26.3\%]), supporting the findings of previous researchers to this result [3,19,20,27-29]. Some studies have verified that these markers are among the radiographic parameters of greatest importance for indicating the risk of IAN damage during removal of the third molar of the mandible where the risks range from $8 \%$ to $22 \%[4,26,27,30]$. However, it is important to note that radiographic markers themselves do not directly predict nerve injury; Instead, they predict the likelihood of seeing the canal / nerve in the extraction socket.

In the current study; the majority (61\%) of 1017 third molars presented radiographic markers of contact between their roots and the mandibular canal. This result is similar to that reported by Dalili et al. Kositbowornchai et al. and Lacerda-Santos et al. [29,30,3]. Nevertheless, several studies discussed the results of radiographic examinations of 1589 affected third molars and reported a low frequency of these markers [16]. This difference in results may be related to the inclusion criteria used by these authors, as only 3 of the seven indicative signs were adopted (deflection of the canal, deflection of the roots, and darkening of the roots) [16].

The depth of the third molar in the mandible, the angle of the teeth, and the position of the roots in relation to the mandibular canal can influence the risk of IAN neurosensory injury [25]. In this study, radiographic signs of contact between the third molar and the lower canal were frequently associated with teeth at the positions IB (90.7\%), IIA (88.4\%), IIC (100\%) and IIIC $(100 \%)$. Consequently, the deeper the tooth is influenced in the ram, the greater the frequency of the marks, a finding that approves the results of a study by Rodriguez et al. And Lacerda-Santos et al $[3,22]$.

A statistically significant association between the presence of radiographic signs of proximity and a mesioangular third molar position was observed in $76 \%$ of our patients. However, the previously mentioned studies found that the incidence of damage to the IAN is greatest after extraction of horizontally impacted third molars and lowest when the extracted tooth was in a vertical position [16]. When we consider the rate of TM / MP in conjunction with the sex of the patient, there was a significant association between TM / MP with female patients as the TM / MP rate was $73 \%$, with an associated odds ratio of 1.5, CI95\%: 1.1-2.1 ( $\mathrm{p}=0.036)$ (Table 2). The present results are in contrast to a study conducted by Tachinami et al. where they discovered a greater rate of TM / MP in male patients [28]. However, our result is comparable to that described by Lacerda-Santos et al. in that an important connection was found between TM / MP with female patients [3]. The current study also had a significant association between TM / MP with the younger age group (15-24 years) as the TM / MP rate was $81 \%$, with an associated odds ratio of $3.2,95 \%$ CI: $2.2-4.6$ $(\mathrm{p}<0.0001)$. (Table 2$)$, this result is similar to that reported by Lacerda-Santos et al. [3]; Sarikov and Juodzbalys [16] and Kim et al. [25] found that more than half of the patients who provided contact with the third molar with the maxillary canal were 24 years of age or younger. However, these data are not worrisome, because other researchers have noted that young patients are less likely to experience permanent damage to IAN after a third molar extraction. [16,25].

The present results demonstrate the importance of panoramic radiography as a preoperative screening examination for removal of the third molars of the lower jaw. A review of the literature did not find any studies indicating that the preoperative use of cone beam computed tomography significantly reduces the incidence of nerve damage during third molar surgery [31]. Despite the consequences of the imaging examination used, if radiographic signs of proximity are present, the physician should adopt surgical techniques that facilitate the removal of the third molar while minimizing the risk of damage to the IAN: cutting teeth, cutting the bone, avoiding sudden movements of elevators, and avoiding the use of forceps. 


\section{Conclusion}

This study was designed to examine the relationship between the third molars and the lower canal in relation to variables such as the patient's sex and age, tooth position, and radiographic parameters. This information can inform the doctor about surgical planning, helping to avoid damage to the IAN. The frequency near the molar duct / mandible was greater in female patients and patients aged 24 years or younger. The most common signs of proximity were darkening of the roots and discontinuity of the mandibular canal. The tooth positions most associated with the proximity of the third molar to the lower canal were the $\mathrm{C}$ and the mesioangular position.

\section{Author contribution}

Prof. Dr. Nashwan Yahya Al-Shamahi, Professor of Radiology, Faculty of Medicine and Health Sciences, examined the panoramic radiographs to assess signs of proximity with Dr. Al-Kasem M Abbas, Professor of Dental Surgery, Faculty of Dentistry, Sana'a University. Other authors analyzed the data and wrote the manuscript, and reviewed it.

\section{Acknowledgments}

This work was supported by Sana'a University, Sana'a, Yemen.

\section{Conflict Of Interest}

"No conflict of interest associated with this work".

\section{References}

1. Alhadi Y, Al-Shamahy HA, Aldilami A, Al-Hamzy M, AlHaddad KA, et al (2019). Prevalence and Pattern of Third Molar Impaction in Sample of Yemeni Adults. On J Dent \& Oral Health, 1(5):1-5. OJDOH.MS.ID.000524. DOI: 10.33552/OJDOH.2019.01.000524.

2. Alhadi, Y, Rassem A H, Al-Shamahy HA, and Al-Ghaffari KM (2019). Causes for extraction of permanent teeth in general dental practices in Yemen. Universal Journal of Pharmaceutical Research, 4(2):1-6. doi:https://doi. org/10.22270/ujpr.v4i2.249.

3. Lacerda-Santos JT, Granja GL, Vasconcelos Catão MH et al., (2020). Signs of the proximity of third molar roots to the mandibular canal: an observational study in panoramic radiographs. General dentistry, 68(2):30-35.

4. Lauesen SR, Andreasen JO, Gerds TA, Christensen SS, Borum M, Hillerup S (2013). Association between third mandibular molar impaction and degree of root development in adolescents. Angle Orthod, 83(1):3-9.

5. Li ZB, Qu HL, Zhou LN, Tian BM, Gao LN, Chen FM (2017). Nonimpacted third molars affect the periodontal status of adjacent teeth: a cross-sectional study. J Oral Maxillofac Surg, 75(7):1344-1350.

6. Nakamori K, Tomihara K, Noguchi M (2014). Clinical significance of computed tomography assessment for third molar surgery. World J Radiol, 6(7):417-423.
7. Huang CK, Lui MT, Cheng DH (2015). Use of panoramic radiography to predict postsurgical sensory impairment following extraction of impacted mandibular third molars. J Chin Med Assoc, 78(10):617-622.

8. Pedro FL, Bandéca MC, Volpato LE, et al., (2014). Prevalence of impacted teeth in a Brazilian subpopulation. J Contemp Dent Pract, 15(2):209-213.

9. Syed KB, Alshahrani FS, Alabsi WS, et al., (2017). Prevalence of distal caries in mandibular second molar due to impacted third molar. J Clin Diagn Res, 11(3):ZC28ZC30.

10. Al-Zoubi H, Alharbi AA, Ferguson DJ, Zafar MS (2017). Frequency of impacted teeth and categorization of impacted canines: a retrospective radiographic study using orthopantomograms. Eur J Dent, 11(1):117-121.

11. Ayaz H, Rehman A(2012). Pattern of impacted mandibular third molar in patients reporting to Department of Oral and Maxillofacial Surgery, Khyber College of Dentistry, Peshawar. J Khyber Coll Dent (JKCD), 2(2):50-53.

12. Santosh P (2015). Impacted mandibular third molars: review of literature and a proposal of a combined clinical and radiological classification. Ann Med Health Sci Res, $5(4): 229-234$.

13. Oenning AC, Melo SL, Groppo FC, Haiter-Neto F (2015). Mesial inclination of impacted third molars and its propensity to stimulate external root resorption in second molars: a cone-beam computed tomographic evaluation. $J$ Oral Maxillofac Surg, 73(3):379-386.

14. Miranda da Rosa F, Oliveira MG, Palmeira da Silva V, Rados PV, Sant'Ana Filho M (2015). Relationship between the positions of impacted third molars and the presence of dentigerous cysts. Gen Dent, 63(4):43-46.

15. Gisakis IG, Palamidakis FD, Farmakis ET, Kamberos G, Kamberos S (2011). Prevalence of impacted teeth in a Greek population. J Investig Clin Dent, 2(2):102-109.

16. Sarikov R, Juodzbalys G (2014). Inferior alveolar nerve injury after mandibular third molar extraction: a literature review. J Oral Maxillofac Res, 5(4):e1.

17. Giovacchini F, Paradiso D, Bensi C, Belli S, Lomurno G, Tullio A (2018). Association between third molar and mandibular angle fracture: a systematic review and metaanalysis. J Craniomaxillofac Surg, 46(4):558-565.

18. Martin A, Perinetti G, Costantinides F, Maglione M (2015). Coronectomy as a surgical approach to impacted mandibular third molars: a systematic review. Head Face Med, 11:9.

19. Rood JP, Shehab BA (1990). The radiological prediction of inferior alveolar nerve injury during third molar surgery. Br J Oral Maxillofac Surg, 28(1):20-25.

20. Tantanapornkul W, Mavin D, Prapaiphittayakun J, Phipatboonyarat N, Julphantong W (2016). Accuracy of 
panoramic radiograph in assessment of the relationship between mandibular canal and impacted third molars. Open Dent J, 10:322-329.

21. Goyal S, Verma P, Raj SS (2016). Radiographic evaluation of the status of third molars in Sriganganagar population - a digital panoramic study. Malays J Med Sci, 23(6):103112.

22. Rodriguez y Baena R, Beltrami R, Tagliabo A, Rizzo S, Lupi SM (2017). Differences between panoramic and cone beam-CT in the surgical evaluation of lower third molars. J Clin Exp Dent, 9(2):e259-e265.

23. Pell GJ, Gregory GT (1933). Impacted mandibular third molars: classification and modified technique for removal. Dent Dig, 39(9):330-338.

24. Winter GB (1926). Principles of Exodontia as Applied to the Impacted Mandibular Third Molar. St Louis: American Medical Book.

25. Kim JW, Cha IH, Kim SJ, Kim MR (2012). Which risk factors are associated with neurosensory deficits of inferior alveolar nerve after mandibular third molar extraction? $J$ Oral Maxillofac Surg, 70(11):2508-2514.

26. Carter K, Worthington S (2016). Predictors of third molar impaction: a systematic review and metaanalysis. J Dent Res, 95(3):267-276.

27. Liu W, Yin W, Zhang R, Li J, Zheng Y (2015). Diagnostic value of panoramic radiography in predicting inferior alveolar nerve injury after mandibular third molar extraction: a meta-analysis. Aust Dent J, 60(2):233-239.

28. Tachinami H, Tomihara K, Fujiwara K, Nakamori K, Noguchi M (2017). Combined preoperative measurement of three inferior alveolar canal factors using computed tomography predicts the risk of inferior alveolar nerve injury during lower third molar extraction. Int J Oral Maxillofac Surg, 46(11):1479-1483.

29. Dalili Z, Mahjoub P, Sigaroudi AK (2011). Comparison between cone beam computed tomography and panoramic radiography in the assessment of the relationship between the mandibular canal and impacted class $\mathrm{C}$ mandibular third molars. Dent Res J (Isfahan), 8(4):203- 210.

30. Kositbowornchai S, Densiri-aksorn W, Piumthanaroj P (2010). Ability of two radiographic methods to identify the closeness between the mandibular third molar root and the inferior alveolar canal: a pilot study. Dentomaxillofac Radiol, 39(2):79-84.

31. Palma-Carrió C, García-Mira B, Larrazabal-Morón C, Peñarrocha-Diago M (2010). Radiographic signs associated with inferior alveolar nerve damage following lower third molar extraction. Med Oral Patol Oral Cir Bucal, 15(6):e886-e890.

Copyright: $(2020$ Hassan Abdulwahab Al-Shamahy. This is an open-access article distributed under the terms of the Creative Commons Attribution License, which permits unrestricted use, distribution, and reproduction in anymedium, provided the original author and source are credited. 